\title{
On the Mahler measure of the composition of two polynomials
}

by

\author{
G. Rhin (Metz) and C. J. SмYтн (Edinburgh)
}

To Ian Cassels on his 75th birthday

1. Introduction. Let $P(x)$ and $T(x)$ be polynomials with integer coefficients, and $P$ irreducible. The aim of this paper is to study the absolute Mahler measure of the composition $P(T(x))$. Recall that the absolute Mahler measure of a polynomial $R(x):=r \prod_{i=1}^{d}\left(x-\gamma_{i}\right)$ is defined to be

$$
\mathfrak{M}(R(x)):=\left(|r| \prod_{i=1}^{d} \max \left(1,\left|\gamma_{i}\right|\right)\right)^{1 / d} .
$$

Also, denote by $\|R\|$ the sum of the absolute values of the coefficients of $R$ (its length).

Our main result is the following:

TheOrem 1. Let $T(x) \in \mathbb{Z}[x]$ be of degree $t \geq 2$, and be divisible by $x$, but $\neq \pm x^{t}$. Then there is a constant $c_{T}>1$ such that for any irreducible polynomial $P(x) \in \mathbb{Z}[x]$, of degree at least 2 , the absolute Mahler measure of $P(T(x))$ satisfies $\mathfrak{M}(P(T(x))) \geq c_{T}$. In fact, $c_{T}$ can be taken to be

$$
c_{T}:=\min \left(1+\frac{1}{2 t\left(2\left\|T^{\prime}\right\|+t+3\right)}, \mathfrak{M}\left(P_{i}(T(x))\right)(i=1, \ldots, N)\right)>1 .
$$

Here the polynomials $P_{i}(i=1, \ldots, N)$, whose degrees total at most $2 t-2$, are the minimal polynomials of the algebraic numbers $T\left(\alpha_{i}\right)$ of degree at least 2 , where $\alpha_{1}, \ldots, \alpha_{N}$ are a complete non-conjugate set of roots of $T(z) T(1 / z)$ $=1$.

The theorem generalises a result of Zhang [Zh], who proved the theorem in the special case of $T(x)=x^{2}-x$. Furthermore, Zagier [Za] proved that the best value of $c_{x^{2}-x}$ is $\left(\frac{1}{2}(1+\sqrt{5})\right)^{1 / 4}$. [In fact, Zhang and Zagier considered $\mathfrak{M}\left(P_{1}(x)\right) \mathfrak{M}\left(P_{1}(1-x)\right)$. Now $P_{1}(x) P_{1}(1-x)=P\left(x^{2}-x\right)$ for some 
polynomial $P$, so that

$$
\mathfrak{M}\left(P_{1}(x)\right) \mathfrak{M}\left(P_{1}(1-x)\right)=\mathfrak{M}\left(P_{1}(x) P_{1}(1-x)\right)^{2}=\mathfrak{M}\left(P\left(x^{2}-x\right)\right)^{2} .
$$

Conversely, $\mathfrak{M}\left(P\left(x^{2}-x\right)\right)^{2}=\mathfrak{M}\left(P\left(x^{2}-x\right)\right) \mathfrak{M}\left(P\left((1-x)^{2}-(1-x)\right)\right)$.]

If we specify that not only $P(x)$ but also $P(T(x))$ be irreducible, then we can obtain a (usually larger) lower bound for $\mathfrak{M}(P(T(x)))$, which moreover does not involve any exceptional values of unknown size (like the $\mathfrak{M}\left(P_{i}(T(x))\right)$ above):

Theorem 2. Let $T[x] \in \mathbb{Z}[x]$ be of degree $t \geq 2$, and be divisible by $x$, but $\neq \pm x^{t}$, and let $P(x) \in \mathbb{Z}[x]$, of degree at least 2 , be such that $P(T(x))$ is irreducible. Then

$$
\mathfrak{M}(P(T(x))) \geq c_{T}^{*}:=1+\frac{1}{2\left(t-t_{0}+4 t\|T\|\right)},
$$

where $x^{t_{0}}$ is the highest power of $x$ dividing $T$.

Notes on the theorems

1. Since $P(T(x))=P_{1}( \pm(T(x)-T(0)))$ for $P_{1}(y)=P( \pm y+T(0))$ it is no restriction to assume that $T(x)$ is divisible by $x$, and has positive leading coefficient.

2. The theorems are clearly false if $T(x)= \pm x^{t}$. If $T(x)$ has leading coefficient $a \geq 1$, then $P(T(x))$ has leading coefficient of modulus at least $a^{p}$, where $p$ is the degree of $P$. Hence, if $a \geq 2$, then $\mathfrak{M}(P(T(x))) \geq|a|^{1 / t}>$ $\max \left(c_{T}, c_{T}^{*}\right)$. We can therefore assume, in the proofs of both theorems, that $T$ is monic.

3. For $P$ linear, $\mathfrak{M}(P(T(x)))=1$ iff $\pm P(y)=y+\varepsilon$ for $\varepsilon \in\{-1,0,1\}$ and $T(x)+\varepsilon= \pm x^{l} C(x)$ with $C(x)$ cyclotomic. Excluding these cases but including all other linear $P$ we then have, under the other conditions of Theorem 1, $\mathfrak{M}(P(T(x))) \geq c_{T}^{\prime}$, where

$$
c_{T}^{\prime}:=\min \left(c_{T}, \mathfrak{M}(T(x)), \mathfrak{M}(T(x)+1), \mathfrak{M}(T(x)-1)\right) .
$$

The proof of Theorem 2 does not work if $P$ is linear, as Lemma 6 cannot be applied.

4. The constants $c_{T}$ and $c_{T}^{*}$ can be improved, at the expense of some complication. For instance, $c_{T}^{*}$ can be taken to be $\max \left(\lambda^{\prime}, \lambda^{\prime \prime}\right)$, where $\lambda^{\prime}$ is the root $>1$ of $\lambda^{-8}+\lambda^{-2\left(2 t-t_{0}\right)} / l_{1}=1$ and $\lambda^{\prime \prime}$ is the root $>1$ of $\lambda^{-8}+$ $\lambda^{-2\left(t-t_{0}\right)} / \max \left(l_{0}, l_{1}\right)=1$. Here $l_{0}=\left\|\left(x^{t} T(1 / x)\right)^{\prime}\right\|$ and $l_{1}=\left\|T^{\prime}\right\|$. Further improvements in $c_{T}$ and $c_{T}^{*}$ can usually be made, using the details of the proofs, for specific $T$.

2. Background. The results of this paper can be regarded as one of a series in which a lower bound is found for the mean value, over the conjugates of an algebraic number $\alpha$, of some function. To obtain non-trivial 
bounds, one must of course use the fact that these conjugates are not arbitrary complex numbers. This is usually done by choosing a symmetric function of the conjugates which is a non-zero integer. For instance, Siegel $[\mathrm{Si}]$, in bounding the trace of a totally positive algebraic integer, used the discriminant of $\alpha$. Schinzel and Zassenhaus [ScZas], and later Blanksby and Montgomery [BlMo], in connection with Lehmer's question, used the resultant of $\alpha$ and a root of unity. Cassels [Ca], bounding the maximum modulus of the conjugates of a non-reciprocal algebraic integer $\alpha$, used the resultant of $\alpha$ and $1 / \alpha$. Dobrowolski [Do], again in connection with Lehmer's question, used the resultant of $\alpha$ and $\alpha^{p}$, for $p$ prime. The papers [Sm1], [Sm2], [RhSm], [Fl1], [Fl2], on the spectra of the mean values of various functions $f(x)$ over conjugate sets of algebraic integers, used inequalities of the form

$$
f(x)-\sum_{j} a_{j} \log \left|P_{j}(x)\right| \geq c>0,
$$

where the $P_{j}$ are minimal polynomials of $\alpha^{\prime}$ with $f\left(\alpha^{\prime}\right)$ small, and the $a_{j}$ are $>0$. The resultants of $\alpha$ and $\alpha^{\prime}$ are assumed not to vanish. Then it follows easily that the required mean value is at least $c$, except possibly for $\alpha$ conjugate to some $\alpha^{\prime}$. This often yields a spectrum of the smallest mean values. In 1993 Zagier [Za], in connection with $\mathfrak{M}\left(P\left(x^{2}-x\right)\right)$, introduced a fruitful extension of (2.1), by producing inequalities of this type with $\left|P_{j}(x)\right|$ replaced by $\left|P_{j}(x)\right|_{\nu}$, for each valuation $\nu$ of a field containing $\alpha$. This enabled him to readily treat means over conjugate sets of (not necessarily integer) algebraic numbers.

Very recently, Beukers and Zagier [BeZa] have made further substantial improvements in this area, making possible a much wider class of lower bounds for heights of certain algebraic points on varieties. They do this by working over products of projective spaces $P^{n}(\overline{\mathbb{Q}})$ over the algebraic numbers $\overline{\mathbb{Q}}$. This makes the optimisation of auxiliary functions technically much easier. One reason is that all variables can be assumed to be of modulus at most 1. Further, when optimising over a hypersurface, considerations of harmonicity enable one to assume that at most one variable has modulus strictly smaller than 1 .

3. Results of Beukers and Zagier. In this section we state a version of Lemma 3.1 of [BeZa] (Lemma 3). We state only a special case, which is sufficient for our applications. We also state an important result (Proposition 4), which they derive from that lemma. We use Lemma 3 to prove Theorem 1, after some optimisation. Theorem 2 follows from Proposition 4, the optimisation having already been carried out in the proof of Proposition 4. 
We first need some notation, essentially that from $[\mathrm{BeZa}]$. Let $\mathbb{P}(\overline{\mathbb{Q}})$ denote the projective line over $\overline{\mathbb{Q}}$, with

$$
x=\left(x_{10}, x_{11}, x_{20}, x_{21}, \ldots, x_{t 0}, x_{t 1}\right)
$$

a typical point of $\mathbb{P}(\overline{\mathbb{Q}})^{t}$. Let $X(\overline{\mathbb{Q}})$ be a hypersurface in $\mathbb{P}(\overline{\mathbb{Q}})^{t}$ with equation $F(x)=0$ having integer coefficients, and let $X_{1}$ denote the intersection of $X$ with the polydisc $\left\{\left|x_{i j}\right| \leq 1, i=1, \ldots, t, j=0,1\right\}$. Let $G(x)$ be a multihomogeneous polynomial over $\mathbb{P}(\mathbb{Q})^{t}$, of degree $d_{i}$ in $x_{i}=\left(x_{i 0}, x_{i 1}\right)(i=$ $1, \ldots, t)$. To define the height $H(\alpha)$ of $\alpha$ in an algebraic number field $K$ of degree $D=[K: \mathbb{Q}]$ over $\mathbb{Q}$, we let ||$_{\nu}$ be the valuations of $K$, with completions $K_{\nu}$ of degrees $D_{\nu}=\left[K_{\nu}: \mathbb{Q}_{\nu}\right]$ over $\mathbb{Q}_{\nu}$. For archimedean $\nu$ put $|x|_{\nu}=|x|^{-D_{\nu} / D}$, while for $\nu$ non-archimedean normalise ||$_{\nu}$ so that $|p|_{\nu}=p^{-D_{\nu} / D}$ for the unique rational prime $p$ with $|p|_{\nu}<1$. Then define $H(\alpha)=\prod_{\nu} \max \left(1,|\alpha|_{\nu}\right)$, while for $\alpha=\left(\alpha_{0}, \alpha_{1}\right) \in \mathbb{P}(\overline{\mathbb{Q}})$ put $H(\alpha)=$ $\prod_{\nu} \max \left(\left|\alpha_{0}\right|_{\nu},\left|\alpha_{1}\right|_{\nu}\right)$. These definitions are independent of the choice of the field $K$ containing $\alpha$.

Lemma 3 (Special case of Lemma 3.1 of [BeZa]). Let $\Lambda=\max _{x \in X_{1}}|G(x)|$. Then for any point $x \in X(\overline{\mathbb{Q}})$ with $G(x) \neq 0$ we have

$$
\prod_{i=1}^{t} H\left(x_{i}\right)^{d_{i}} \geq 1 / \Lambda .
$$

The lemma gives us a lower bound for the height of a point which is on the hypersurface $F=0$ but not on $G=0$. Of course, only if $\Lambda<1$ does the lemma give a non-trivial lower bound.

Now let $F$ be a bihomogeneous polynomial in $x_{i}=\left(x_{i 0}, x_{i 1}\right)(i=1,2)$ over $\overline{\mathbb{Q}}$, of bidegrees $d_{i}$ in $x_{i}$ and degrees $d_{i j}$ in $x_{i j} \quad(i=1,2 ; j=0,1)$. Let $E$ be a subset of $\{(1,0),(2,0)\}$, and put

$$
c_{F}=\max _{(i, j) \notin E}\left\|\frac{\partial F}{\partial x_{i j}}\right\|
$$

while for $i=1,2$,

$$
\delta_{i}= \begin{cases}d_{i 1}-\left(d_{i}-d_{i 0}\right) / 2 & \text { if }(i, 0) \in E, \\ \left(d_{i 0}+d_{i 1}-d_{i}\right) / 2 & \text { if }(i, 0) \notin E,\end{cases}
$$

and $\delta=\max \left(\delta_{1}, \delta_{2}\right)$. For a hypersurface $F(x)=0$, let $F\left(x^{-1}\right)=0$ denote the hypersurface $F\left(x_{11}, x_{10}, x_{21}, x_{20}\right)=0$. Then

Proposition 4 ([BeZa]). Let $\varrho$ be the unique real root larger than 1 of $x^{-2}+c_{F}^{-1} x^{-\delta}=1$. Then for each point $x$ on $F(x)=0$ but not on $x_{10} x_{11} x_{20} x_{21} F\left(x^{-1}\right)=0$ we have

$$
H\left(x_{1}\right) H\left(x_{2}\right) \geq \varrho^{1 / 2} .
$$


4. Preliminary lemmas. We need the following lemma:

Lemma 5. Let $T(x) \in \mathbb{Q}[x]$, of degree $t$, be divisible by $x$, and be such that $T(x)=\beta$ and $T(1 / x)=\beta^{\prime}$ have the same roots, with the same multiplicities. Suppose further that $\beta^{\prime}$ is irrational. Then $T(x)= \pm \sqrt{\beta \beta^{\prime}} x^{t}$.

Pr o of. Let $a \neq 0$ be the leading coefficient of $T$. Then

$$
\beta^{\prime}(T(x)-\beta)=a\left(\beta^{\prime} x^{t}-x^{t} T(1 / x)\right),
$$

identically in $x$. Note that $x^{t} T(1 / x)$ has degree at most $t-1$. Now, on comparing coefficients of $x, x^{2}, \ldots, x^{t-1}$ we see, from the irrationality of $\beta^{\prime}$, that these coefficients must all be 0 . Then we get $a^{2}=\beta \beta^{\prime}$ on putting $x=0$.

Using this result, we can prove the following

Lemma 6. Suppose that $P(x), T(x) \in \mathbb{Z}[x]$, where $P$ has degree $p \geq 2$ and $T(x)$, of degree $t$, is divisible by $x$ but not by $x^{t}$. Suppose further that $P(T(x))$ is irreducible over $\mathbb{Q}$, with $\alpha$ a root of $P(T(x))=0$. Then there is a conjugate $\alpha^{\prime}$ of $\alpha$ with $T\left(1 / \alpha^{\prime}\right) \neq T(1 / \alpha)$.

Proof. Put $\beta=T(\alpha), \beta^{\prime}=T(1 / \alpha)$. Then $\alpha$ is a root both of $T(x)=\beta$ and $T(1 / x)=\beta^{\prime}$. Note that $\beta$ is of degree $p$ over $\mathbb{Q}$, since by the irreducibility of $P(T(x)), P(x)$ is certainly irreducible.

Now suppose that $T\left(1 / \alpha^{\prime}\right)=T(1 / \alpha)$ for each root $\alpha^{\prime}$ of $T(x)=\beta$. Then $T(1 / \alpha)$ is in the fixed field of $\operatorname{Gal}(\mathbb{Q}(\alpha) / \mathbb{Q}(\beta))$, i.e. $\beta^{\prime}=T(1 / \alpha) \in \mathbb{Q}(\beta)$. But now both $T(x)=\beta$ and $T(1 / x)=\beta^{\prime}$ are essentially the minimal polynomials of $\alpha$ over $\mathbb{Q}(\beta)$, so have the same roots. Also $\left[\mathbb{Q}\left(\beta^{\prime}\right): \mathbb{Q}\right]=[\mathbb{Q}(\beta): \mathbb{Q}]=p$ $\geq 2$, so that $\beta^{\prime}$ is irrational. Then Lemma 5 gives a contradiction.

Lemma 7. Let $P(x), T(x) \in \mathbb{Z}[x]$ with $P$ irreducible, of degree at least 2 , and $T$ of degree $t$, divisible by $x$ but not by $x^{t}$. Then $P(T(x))$ is not cyclotomic, and $\mathfrak{M}(P(T(x)))>1$.

Proof. Suppose that $P(T(x))$ is cyclotomic. Then, for any zero $\beta$ of $P$, $T(x)=\beta$ has all roots being roots of unity, so that $T(x)-\beta=a \prod_{i}\left(x+\theta_{i}\right)$, where $\left|\theta_{i}\right|=1$. From this, $T(1 / x)-\bar{\beta}=\bar{a} x^{-t}\left(\prod_{i} \theta_{i}\right)^{-1} \prod_{i}\left(x+\theta_{i}\right)$, so that $T(x)=\beta$ and $T(1 / x)=\bar{\beta}$ have the same roots. Now Lemma 5 gives a contradiction. Finally, $\mathfrak{M}(P(T(x)))>1$ using a classical result of Kronecker to the effect that the only polynomials in $\mathbb{Z}[x]$ with measure 1 are those of the form $\pm x^{l} C(x), C$ cyclotomic.

Lemma 8. For any $T(z) \in \mathbb{C}[z]$ of degree $t$ we have, for $z \in \mathbb{C} \backslash\{0\}$,

$$
|T(z) T(1 / z)-1| \leq\left.|| T(z)\right|^{2}-1\left|+l_{1}\right| T(z) \mid m^{t}\left(1-m^{-2}\right) .
$$

Here $l_{1}=\left\|T^{\prime}(z)\right\|$ and $m=\max \left(|z|,|z|^{-1}\right)$.

Proof. We use the inequality

$$
|T(x)-T(y)| \leq l_{1}|x-y|(\max (1,|x|,|y|))^{t-1} \quad(x, y \in \mathbb{C})
$$


which is easily checked. Then, for $x=\bar{z}, y=1 / z$, the lemma follows immediately from

$$
|T(z) T(1 / z)-1|=|T(z) T(\bar{z})-1-T(z)(T(\bar{z})-T(1 / z))| .
$$

5. Proof of Theorem 1. For the proof, we apply Lemma 3 with

$$
F(x):=\left(T\left(\frac{x_{11}}{x_{10}}\right)+\prod_{i=1}^{t}\left(\frac{-x_{i 1}}{x_{i 0}}\right)\right) x_{10}^{t} \prod_{i=2}^{t} x_{i 0}
$$

and

$$
G(x):=\left(\prod_{i=1}^{t} x_{i 0} x_{i 1}\right)^{B+t}\left(T\left(\frac{x_{11}}{x_{10}}\right) T\left(\frac{x_{10}}{x_{11}}\right)-1\right),
$$

where $B$ is an integer to be chosen later.

Let $P$ be irreducible of degree $p$, with $P(\beta)=0$, and suppose that $T(x)-\beta$ splits over $\mathbb{Q}(\beta)$ into irreducible factors $\prod_{i=1}^{L} T_{i}(x)$. Let $\alpha_{i 1}, \ldots, \alpha_{i t_{i}}$ be the zeros of $T_{i}(i=1, \ldots, L)$. Then since $\alpha_{i j}$ and $\alpha_{i j^{\prime}}$ are conjugate over $\mathbb{Q}$, and $\left[\mathbb{Q}\left(\alpha_{i 1}\right): \mathbb{Q}\right]=t_{i} p$, we have

$$
\prod_{i=1}^{L} \prod_{j=1}^{t_{i}} H\left(\alpha_{i j}\right)=\prod_{i=1}^{L} H\left(\alpha_{i 1}\right)^{t_{i}}=\mathfrak{M}(P(T(x)))^{t} .
$$

Next, rename the $\alpha_{i j}$ as $\alpha_{1}, \ldots, \alpha_{t}$. Then $T(x)-\beta=\prod_{i=1}^{t}\left(x-\alpha_{i}\right)$, since $T(x)$ is assumed monic (see Note 2 of Section 1 ), so that $\beta=T\left(\alpha_{1}\right)=$ $-\prod_{i}\left(-\alpha_{i}\right)$. Hence $x=\left(\alpha_{1}, 1, \alpha_{2}, 1, \ldots, \alpha_{t}, 1\right)$ lies on $F=0$. Now $P\left(T\left(\alpha_{1}\right)\right)$ $=0$, i.e. $P$ is the minimal polynomial of $T\left(\alpha_{1}\right)$, so that $G(x)=0$ only if $P$ is the minimal polynomial of some $T\left(\alpha_{1}\right)$, where $\alpha_{1}$ is a root of $T\left(\alpha_{1}\right) T\left(1 / \alpha_{1}\right)$ $=1$. Since $x^{t-1}(T(x) T(1 / x)-1) \in \mathbb{Z}[x]$ has degree at most $2 t-2$, the sum of the degrees of the minimal polynomials $P_{i}(x)(i=1, \ldots, N)$ of all $T\left(\alpha_{1}\right)$ with $T\left(\alpha_{1}\right) T\left(1 / \alpha_{1}\right)=1$ is also at most $2 t-2$. So $G(x) \neq 0$ unless $P=P_{i}$ for some $i$. Since these $P_{i}$ are accounted for in the statement of the theorem, we can assume that $G(x) \neq 0$. Note that the $\mathfrak{M}\left(P_{i}(T(x))\right)$ are $>1$ by Lemma 7.

Now, in the notation of Section $3, d_{i}=2(B+t)$, so, on applying Lemma 3 , we obtain

$$
\mathfrak{M}(P(T(x)))=\left(\prod_{i=1}^{t} H\left(\alpha_{i}\right)^{2(B+t)}\right)^{1 /(2 t(B+t))} \geq \Lambda^{-1 /(2 t(B+t))} .
$$

It remains to estimate this lower bound, and to choose $B$ so that it is as large as possible. As noted in the proof [BeZa] of Proposition 4, this maximum will occur at a point where at most one of the $x_{i}$ is less than one in modulus, with all other $\left|x_{i j}\right|=1$. Essentially this is because there is one constraint $F(x)=0$ on the $x_{i j}$. We consider the four possibilities: 
1. $\left|x_{10}\right| \leq 1$. Put $x_{10}=x, x_{11}=\omega$, so that, on $X, T(\omega / x)=\varrho / x$, where $|\omega|=|\varrho|=1$. Then using Lemma 8 ,

$$
\begin{aligned}
|G| & =|x|^{B+t}|T(\omega / x) T(x / \omega)-1| \\
& \leq|x|^{B+t}\left\{\left(|x|^{-2}-1\right)+l_{1}|x|^{-1}\left(1-|x|^{2}\right)|x|^{-t}\right\} \\
& =\left(1-|x|^{2}\right)|x|^{B-1}\left\{|x|^{t-1}+l_{1}\right\} .
\end{aligned}
$$

2. $\left|x_{11}\right| \leq 1$. Put $x_{11}=x, x_{10}=\omega$, so that $T(x / \omega)=\varrho x$, where $|\omega|=$ $|\varrho|=1$. Then (5.3) again holds, and, in a similar way to (5.4) we get

$$
|G| \leq\left(1-|x|^{2}\right)|x|^{B+1}\left\{|x|^{t-1}+l_{1}\right\} .
$$

3. $\left|x_{i 0}\right| \leq 1, i>1$. Then we have similarly $x_{i 0}=x, T(\omega)=\varrho / x$, and

$$
|G|=|x|^{B+t}|T(\omega) T(1 / \omega)-1|=\left(1-|x|^{2}\right)|x|^{B+t} .
$$

4. $\left|x_{i 1}\right| \leq 1, i>1$. Then $x_{i 1}=x, T(\omega)=\varrho x$ and

$$
|G|=|x|^{B+t}|T(\omega) T(1 / \omega)-1|=\left(1-|x|^{2}\right)|x|^{B+t} .
$$

We see, therefore, that (5.4) of case 1 gives the largest upper bound for $|G|$.

Now for $A>0$,

$$
\max _{y \in[0,1]}\left(1-y^{2}\right) y^{2 A}=\frac{A^{A}}{(A+1)^{A+1}}=\frac{1}{A}\left(1-\frac{1}{A+1}\right)^{A+1}<\frac{1}{e A} .
$$

Hence, from (5.4),

$$
\Lambda \leq \frac{2}{e}\left\{\frac{1}{B+t-2}+\frac{l_{1}}{B-1}\right\} \leq \frac{2\left(1+l_{1}\right)}{e(B-1)} .
$$

Now, choosing $B=2 l_{1}+3,(5.2)$ gives

$$
\mathfrak{M}(P(T(x))) \geq e^{1 /\left(2 t\left(2 l_{1}+t+3\right)\right)}>1+\frac{1}{2 t\left(2 l_{1}+t+3\right)} .
$$

6. Proof of Theorem 2. Take

$$
F\left(x_{10}, x_{11}, x_{20}, x_{21}\right)=x_{10}^{t} x_{20}^{t}\left(T\left(\frac{x_{11}}{x_{10}}\right)-T\left(\frac{x_{21}}{x_{20}}\right)\right) .
$$

Assume that $P(T(x))$ is irreducible, with $P(T(\alpha))=0$. Then, by Lemma 6 , there is another zero $\alpha^{\prime}$ of $P(T(x))$ with $T\left(1 / \alpha^{\prime}\right) \neq T(1 / \alpha)$. So the point $\left(\alpha, 1, \alpha^{\prime}, 1\right)$ is on $F(x)=0$ but not on $F\left(x^{-1}\right)=0$. Thus, applying Proposition 4 , and using the fact that $H(\alpha, 1)=H\left(\alpha^{\prime}, 1\right)=H(\alpha)=\mathfrak{M}(P(T(x)))$, we get

$$
\mathfrak{M}(P(T(x))) \geq \varrho^{1 / 4}
$$


To calculate $\varrho$, first note that $\left\|\partial F / \partial x_{1 j}\right\|=\left\|\partial F / \partial x_{2 j}\right\|=l_{j}(j=0,1)$, in the notation of Note 4 of Section 1 . Then

$$
c_{F}= \begin{cases}l_{1} & \text { if } E=\{(1,0),(2,0)\} \\ \max \left(l_{0}, l_{1}\right) & \text { otherwise }\end{cases}
$$

and

$$
\delta= \begin{cases}t-t_{0} / 2 & \text { if } E=\{(1,0),(2,0)\} \\ \frac{1}{2}\left(t-t_{0}\right) & \text { otherwise }\end{cases}
$$

where $x^{t_{0}} \| T(x)$. Let $\varrho^{\prime}$ be the value of $\varrho$ when $E=\{(1,0),(2,0)\}$, and $\varrho^{\prime \prime}$ be the value of $\varrho$ for all other $E$, as defined in Proposition 4 . Then we have $\varrho=\max \left(\varrho^{\prime}, \varrho^{\prime \prime}\right)$, which, with $(6.1)$, gives the estimate of $c_{T}^{*}$ in Note 4 of Section 1 . To find a simpler, slightly smaller lower bound $c_{T}^{*}$, we put $\lambda=\left(\varrho^{\prime \prime}\right)^{1 / 4}$. Then, since $c_{F} \leq \max \left(l_{0}, l_{1}\right) \leq t\|T\|$, and from the definition of $\varrho^{\prime \prime}$,

$$
1-\lambda^{-8}=\lambda^{-2\left(t-t_{0}\right)} / \max \left(l_{0}, l_{1}\right) \geq \lambda^{-2\left(t-t_{0}\right)} /(t\|T\|) .
$$

Putting $\lambda=1+\varepsilon$ and using $\lambda^{-k} \geq 1-k \varepsilon$ we obtain

$$
8 \varepsilon \geq\left(1-2\left(t-t_{0}\right) \varepsilon\right) /(t\|T\|)
$$

so that

$$
c_{T}^{*} \geq \varrho^{1 / 4} \geq\left(\varrho^{\prime \prime}\right)^{1 / 4}=1+\varepsilon \geq 1+\frac{1}{2\left(t-t_{0}+4 t\|T\|\right)} .
$$

Acknowledgements. Theorem 1 was originally proved by the use of an auxiliary function inequality of the form

$$
\log M_{y}(T(y)-T(x))-\frac{1}{2} \log |T(x)|-c_{1} \log \left|x^{t-1}(T(x) T(1 / x)-1)\right| \geq c_{2}
$$

valid for all $x \in \mathbb{C}$, generalising an inequality of Zagier [Za] for $T(x)=x^{2}-x$. Here $c_{1}, c_{2}>0$, and $M_{y}(T(y)-T(x))$, a function of $x$ only, is the classical (relative) Mahler measure of $T(y)-T(x), T(y)-T(x)$ being regarded as a polynomial in $y$. Following a one-day meeting on this and related topics in Paris in May 1996, where Frits Beukers described his and Zagier's new results $[\mathrm{BeZa}]$, we realised that translating our proof into their new framework would significantly improve the lower bound $c_{T}$, and simplify the proof. We thank Marie José Bertin for organising the meeting, and Don Zagier for some useful comments on Theorem 2. We also thank David Boyd for helpful remarks in connection with Lemma 7.

The second author wishes to thank the University of Metz for its hospitality, during which some of this work was done.

\section{References}

[BeZa] F. Beukers and D. Zagier, Lower bounds of heights of points on hypersurfaces, this volume, 103-111. 
[BlMo] P. E. Blanksby and H. L. Montgomery, Algebraic integers near the unit circle, Acta Arith. 18 (1971), 355-369.

[Ca] J. W. S. Cassels, On a problem of Schinzel and Zassenhaus, J. Math. Sci. 1 (1966), 1-8.

[Do] E. Dobrowolski, On a question of Lehmer and the number of irreducible factors of a polynomial, Acta Arith. 34 (1979), 391-401.

[Fl1] V. Flammang, Sur la longueur des entiers algébriques totalement positifs, J. Number Theory 54 (1995), 60-72.

[Fl2] -, Two new points in the spectrum of the absolute Mahler measure of totally positive algebraic integers, Math. Comp. 65 (1996), 307-311.

[RhSm] G. Rhin and C. Smyth, On the absolute Mahler measure of polynomials having all zeros in a sector, ibid. 64 (1995), 295-304.

[ScZas] A. Schinzel and H. Zassenhaus, A refinement of two theorems of Kronecker, Michigan Math. J. 12 (1965), 81-84.

[Si] C. L. Siegel, The trace of totally positive and real algebraic integers, Ann. of Math. 46 (1965), 302-312.

[Sm1] C. J. Smyth, On the measure of totally real algebraic integers II, Math. Comp. 37 (1981), 205-208

[Sm2] - , The mean values of totally real algebraic integers, ibid. 42 (1984), 663-681.

[Za] D. Zagier, Algebraic numbers close to both 0 and 1, ibid. 61 (1993), 485-491.

[Zh] S. Zhang, Positive line bundles on arithmetic surfaces, Ann. of Math. (2) 136 (1992), 569-587.

URA CNRS no.399

Département de Mathématiques Université de Metz

Ile du Saulcy

57045 Metz Cedex 1, France

E-mail: rhin@poncelet.univ-metz.fr
Department of Mathematics and Statistics University of Edinburgh JCMB, King's Buildings Mayfield Road Edinburgh EH9 3JZ, Scotland, UK E-mail: chris@maths.ed.ac.uk 